\title{
On the Use of Discrete Fourier Transform for Solving Biperiodic Boundary Value Problem of Biharmonic Equation in the Unit Rectangle
}

\author{
Agah D. Garnadi
}

31 December 2017

\author{
Department of Mathematics, \\ Faculty of Mathematics and Natural Sciences, \\ Bogor Agricultural University \\ Jl. Meranti, Kampus IPB Darmaga, Bogor, 16680 Indonesia
}

\begin{abstract}
This note is addressed to solving biperiodic boundary value problem of biharmonic equation in the unit rectangle. First, we describe the necessary tools, which is discrete Fourier transform for one dimensional periodic sequence, and then extended the results to 2dimensional biperiodic sequence. Next, we use the discrete Fourier transform 2-dimensional biperiodic sequence to solve discretization of the biperiodic boundary value problem of Biharmonic Equation.

$M S C: 15 \mathrm{~A} 09 ; 35 \mathrm{~K} 35 ; 41 \mathrm{~A} 15 ; 41 \mathrm{~A} 29 ; 94 \mathrm{~A} 12$.

Key words: Biharmonic equation, biperiodic boundary value problem, discrete Fourier transform, Partial differential equations, Interpolation.
\end{abstract}

\section{Introduction}

This note is an extension of a work by Henrici [2] on fast solver for Poisson equation to biperiodic boundary value problem of biharmonic equation. This 
note is organized as follows, in the first part we address the tools needed to solve the problem, i.e. introducing discrete Fourier transform (DFT) follows Henrici's work [2]. The second part, we apply the tools already described in the first part to solve discretization of biperiodic boundary value problem of biharmonic equation in the unit rectangle. The need to solve discretization of biharmonic equation is motivated by its occurence in some applications such as elasticity and fluid flows $[1,4]$, and recently in image inpainting [3]. Note that the work of Henrici [2] on fast solver for biperiodic Poisson equation can be seen as solving harmonic inpainting.

\section{Discrete Fourier Transform}

\subsection{One-dimensional Transform}

Let $n \in \mathrm{N}$ and $\left(x_{k}\right)_{k=-\infty}^{\infty}$ is a bilateral infinite sequence of complex number. Let $\Pi_{n}$ denote a set of bilateral infinite sequences with period $n$, i.e.:

$$
\Pi_{n}=\left\{\mathbf{x}=\left(x_{k}\right)_{k=-\infty}^{\infty} ; x_{k} \in \mathbb{C}, x_{k+n . j}=x_{k}, j \in \mathrm{Z}\right\} .
$$

Define in $\Pi_{n}$ an addition and scalar multiplication by:

$$
\begin{aligned}
\mathbf{x}+\mathbf{y} & =\left(x_{k}+y_{k}\right)_{k=-\infty}^{\infty}, \\
c \mathbf{x} & =\left(c x_{k}\right)_{k=-\infty}^{\infty}, \quad \forall c \in \mathbb{C} .
\end{aligned}
$$

Equipped with these operations, $\Pi_{n}$ is a finite dimensional vector space with $\operatorname{dim}\left(\Pi_{n}\right)=n$.

Denote $\omega_{n}:=\exp (2 \pi \imath / n), i^{2}=-1$. One dimensional discrete Fourier transform, denoted by $\mathcal{F}_{n}$, is defined as follows:

$$
\begin{aligned}
\mathcal{F}_{n}: \Pi_{n} & \rightarrow \Pi_{n} \\
\mathbf{x} & \mapsto \mathbf{y}
\end{aligned}
$$

where

$$
y_{m}=\frac{1}{n} \sum_{k=0}^{n-1} \omega_{n}^{-m k} x_{k}
$$

It is clearly that $y_{m+n}=y_{n}$, holds for all $m$, as $\omega_{n}^{n}=1$. Hence the map $\mathcal{F}_{n}$ is well defined. It is easily to show that $\mathcal{F}_{n}$ is a bijective map, hence invertible. The inverse of $\mathcal{F}_{n}$ is defined as:

$$
\begin{aligned}
\mathcal{F}_{n}^{-1}: \Pi_{n} & \rightarrow \Pi_{n} \\
\mathbf{y} & \mapsto \mathbf{x},
\end{aligned}
$$


where:

$$
x_{r}=\sum_{k=0}^{n-1} \omega_{n}^{m r} y_{r} .
$$

Observe that the inverse has close resemblance with the DFT. Defining the conjugate of DFT $\mathcal{F}_{n}$ by:

$$
\left(\overline{\mathcal{F}}_{n} \mathbf{y}\right)_{k}=\frac{1}{n} \sum_{m=0}^{n-1} \omega_{n}^{m k} y_{m},
$$

where the index $k$ denoting the $k$-th component of the sequence, then we have

$$
\mathcal{F}_{n}^{-1}=n \overline{\mathcal{F}}_{n} .
$$

Another operator that also useful when working with DFT is the reversion operator denoted by $\mathcal{R}$. This operator is defined as follow, for $\mathbf{x} \in \Pi_{n}$,

$$
(\mathcal{R} \mathbf{x})_{m}:=x_{-m} .
$$

A left and right composition with $\mathcal{F}_{n}$, we have the following relations:

$$
\begin{aligned}
\left(\mathcal{R} \circ \mathcal{F}_{n} \mathbf{x}\right)_{m} & =\frac{1}{n} \sum_{k=0}^{n-1} \omega_{n}^{k m} x_{k} \\
& =\left(\overline{\mathcal{F}}_{n}\right) . \\
\left(\mathcal{F}_{n} \circ \mathcal{R} \mathbf{x}\right)_{m} & =-\sum_{k=0}^{n-1} \omega_{n}^{-k m} x_{-k}=-\sum_{k=0}^{n-1} \omega_{n}^{k m} x_{k}, \\
& =\left(\overline{\mathcal{F}}_{n}\right) .
\end{aligned}
$$

From these composition, it is clear that

$$
\mathcal{F}_{n}^{-1}=n \mathcal{F}_{n} \mathcal{R}=n \mathcal{R} \mathcal{F}_{n} .
$$

We introduce Hadamard product of two sequences $\mathbf{x}$ and $\mathbf{y}$ in $\Pi_{n}$, which is a component-wise product:

$$
\mathbf{x} \bullet \mathbf{y}=\left(x_{k} y_{k}\right)_{k=-\infty}^{\infty} .
$$

Also we introduce convolution product of two sequences $\mathbf{x}$ and $\mathbf{y}$ in $\Pi_{n}$ by:

$$
\mathbf{x} * \mathbf{y}=\mathbf{z}=\left(z_{k}\right)_{k=-\infty}^{\infty},
$$

where:

$$
z_{k}=\sum_{k=0}^{n-1} x_{k} y_{k-m}=\sum_{k=0}^{n-1} x_{k-} y_{k} .
$$


Both product relate to each other of as follows. Let $\mathbf{x}$ and $\mathbf{y}$ be two sequences in $\Pi_{n}$, and

$$
\mathbf{u}=\mathcal{F}_{n} \mathbf{x}, \quad \mathbf{v}=\mathcal{F}_{n} \mathbf{y}
$$

By definition,

$$
\left(\mathcal{F}_{n}(\mathbf{x} \bullet \mathbf{y})\right)_{m}=\frac{1}{n} \sum_{k=0}^{n-1} x_{k} y_{k} \omega_{n}^{-k m},
$$

as $y_{k}=\left(\mathcal{F}_{n}^{-1} \mathbf{y}\right)_{k}$, then

$$
\begin{aligned}
\frac{1}{n} \sum_{k=0}^{n-1} x_{k} y_{k} \omega_{n}^{-k m} & =\frac{1}{n} \sum_{k=0}^{n-1} x_{k}\left(\sum_{l=0}^{n-1} \omega_{n}^{l k} v_{l}\right) \omega_{n}^{-k m} \\
& =\sum_{l=0}^{n-1} v_{l}\left(\frac{1}{n} \sum_{k=0}^{n-1} x_{k} \omega_{n}^{-k(m-l)}\right)
\end{aligned}
$$

The last inner sum is obviously equal to $\left(\mathcal{F}_{n} \mathbf{x}\right)_{(m-l)}=u_{m-l}$, hence

$$
\begin{aligned}
\left(\mathcal{F}_{n}(\mathbf{x} \bullet \mathbf{y})\right)_{m} & =\sum_{l=0}^{n-1} v_{l} u_{m-l}=(\mathbf{v} * \mathbf{u})_{m}=(\mathbf{u} * \mathbf{v})_{m} \\
& =\left(\mathcal{F}_{n}(\mathbf{x}) * \mathcal{F}_{n}(\mathbf{y})_{m}\right)
\end{aligned}
$$

As it holds for all $m$, then

$$
\mathcal{F}_{n}(\mathbf{x} \bullet \mathbf{y})=\mathcal{F}_{n}(\mathbf{x}) * \mathcal{F}_{n}(\mathbf{y})
$$

Working on $\mathbf{u}=\mathcal{F}_{n}(\mathbf{x})$ and $\mathbf{v}=\mathcal{F}_{n}(\mathbf{y})$, for convolution form, then

$$
\begin{aligned}
\mathbf{u} * \mathbf{v} & =n^{2} \mathcal{F}_{n}\left(\mathcal{F}_{n}^{-1}(\mathbf{x}) \bullet \mathcal{F}_{n}^{-1}(\mathbf{y})\right) \\
& =n^{2} \mathcal{F}_{n}\left(R \mathcal{F}_{n}(\mathbf{x}) \bullet R \mathcal{F}_{n}(\mathbf{y})\right) \\
& =n^{2} \mathcal{F}_{n}\left(R\left(\mathcal{F}_{n}(\mathbf{x}) \bullet \mathcal{F}_{n}(\mathbf{y})\right)\right) \\
& =n\left(n R \mathcal{F}_{n}\right)\left(\mathcal{F}_{n}(\mathbf{x}) \bullet \mathcal{F}_{n}(\mathbf{y})\right) \\
& =n\left(\mathcal{F}_{n}^{-1}\right)\left(\mathcal{F}_{n}(\mathbf{x}) \bullet \mathcal{F}_{n}(\mathbf{y})\right) \\
\mathcal{F}_{n}(\mathbf{u} * \mathbf{v}) & =n\left(\mathcal{F}_{n}(\mathbf{x}) \bullet \mathcal{F}_{n}(\mathbf{y})\right)
\end{aligned}
$$

Hence for $\mathbf{x}, \mathbf{y} \in \Pi_{n}$

$$
\mathcal{F}_{n}(\mathbf{u} * \mathbf{v})=n\left(\mathcal{F}_{n}(\mathbf{x}) \bullet \mathcal{F}_{n}(\mathbf{y})\right)
$$




\subsection{Two-dimensional Transform}

Let $n \in \mathrm{N}$ and $\mathbf{x}^{(2)}=\left(x_{k_{1} k_{2}}\right)_{k_{1}, k_{2}=-\infty}^{\infty}$ is a two dimensional bilateral infinite sequence of complex number. Let $\Pi_{n}^{(2)}$ denote a set of bilateral infinite sequences with period $n$ on each direction, i.e.

$$
\begin{aligned}
\Pi_{n}^{(2)}= & \left\{\mathbf{x}=\left(x_{k_{1} k_{2}}\right)_{k_{1}, k_{2}=-\infty}^{\infty} ; x_{k_{1} k_{2}} \in \mathbb{C},\right. \\
& \left.x_{k_{1}+n . j, k_{2}}=x_{k_{1} k_{2}}, x_{k_{1}, k_{2}+n . j}=x_{k_{1} k_{2}}, j \in \mathrm{Z}\right\} .
\end{aligned}
$$

Observe that this set of two-dimensional array is isomorph with a set of bilateral infinite sequence of element in $\Pi_{n}$, i.e. $\left\{\left(\mathbf{x}_{k_{2}}\right)_{k_{2}=-\infty}^{\infty} ; \quad \mathbf{x}_{k_{2}} \in \Pi_{n}, \quad \mathbf{x}_{k_{2}+n . j}=\right.$ $\left.\mathbf{x}_{k_{2}}, j \in \mathrm{Z}\right\}$.

Addition, scalar multiplication, Hadamard product, and convolution in $\Pi_{n}$ easily extended to $\Pi_{n}^{(2)}$ by using the fact that $\Pi_{n}^{(2)}$ is a set of bilateral infinite array of element in $\Pi_{n}$.

The 2-dimensional DFT is defined by

$$
\mathbf{y}^{(2)}=\mathcal{F}_{n}^{(2)} \mathbf{x}^{(2)},
$$

where

$$
y_{m_{1} m_{2}}=\frac{1}{n^{2}} \sum_{k_{1}=0}^{n-1} \sum_{k_{2}=0}^{n-1} \omega_{n}^{-\left(m_{1} k_{1}+m_{2} k_{2}\right)} x_{k_{1} k_{2}} .
$$

Rearrangement of the right hand side, we have the following form:

$$
y_{m_{1} m_{2}}=\frac{1}{n} \sum_{k_{1}=0}^{n-1} \omega_{n}^{-m_{1} k_{1}}\left(\frac{1}{n} \sum_{k_{2}=0}^{n-1} \omega_{n}^{-m_{2} k_{2}} x_{k_{1} k_{2}}\right),
$$

Clearly that the 2-dimensional DFT can be seen as a successive of two onedimensional DFT. Hence, the 2-dimensional DFT is a bijective map, so it is invertible. The inverse is given by:

$$
\left(\mathcal{F}_{n}^{(2)}\right)^{-1}=n^{2} \overline{\mathcal{F}}_{n}^{(2)} .
$$

As 2-dimensional DFT on Hadamard product and convolutions of two elements in $\Pi_{n}^{(2)}$, the following are satisfied:

$$
\begin{aligned}
& \mathcal{F}_{n}^{(2)}(\mathbf{x} \bullet \mathbf{y})=\mathcal{F}_{n}^{(2)}(\mathbf{x}) * \mathcal{F}_{n}^{(2)}(\mathbf{y}) \\
& \mathcal{F}_{n}^{(2)}(\mathbf{u} * \mathbf{v})=n^{2}\left(\mathcal{F}_{n}^{(2)}(\mathbf{x}) \bullet \mathcal{F}_{n}^{(2)}(\mathbf{y})\right)
\end{aligned}
$$




\subsection{Applying DFT for solving Biharmonic equations}

Suppose that $S$ is a unit square,

$$
S=\{(\xi, \eta) ; 0 \leq \xi, \eta \leq 1\} .
$$

We are going to solve biharmonic equation:

$$
\Delta^{2} u(x, y)=f(x, y)
$$

in the rectangle $S$, where $f$ is biperiodic on $S$. Furthermore, we restricts $u$ to be biperiodic, i.e. biperiodic boundary value problem.

To solve the problem, we discretize the equation over a discrete grid on $S$. Suppose that on $S$ we construct a uniform grid with constant spacing $h=1 /(2 n)$ on each direction. Let

$$
\xi_{i}=i h ; \quad \eta_{j}=j h \quad i, j=0,1, \cdots, 2 n,
$$

and obtains approximate values $u_{i, j}$ of $u\left(\xi_{i}, \eta_{j}\right)$ by solving linear equation system from thirteen-points finite difference discretization of biharmonic equations, we have the following discrete equation for biharmonic equation ([5])

$$
\begin{aligned}
{\left[20 u_{i, j}\right.} & -8\left(u_{i+1, j}+u_{i-1, j}+u_{i, j+1}+u_{i, j-1}\right)+ \\
& +2\left(u_{i+1, j+1}+u_{i-1, j+1}+u_{i+1, j-1}+u_{i-1, j-1}\right)+ \\
& \left.+\left(u_{i+2, j}+u_{i-2, j}+u_{i, j+1}+u_{i, j-2}\right)\right] \\
& =h^{4} f_{i, j}
\end{aligned}
$$

where $f_{i, j}=: \mathbf{f} \in \in \Pi_{2 n}^{(2)}$. Observe that the left hand side is a convolution of $\mathbf{u} \in \Pi_{2 n}^{(2)}$ with a bilateral sequence $\mathbf{d}=\left(d_{k m}\right)$ with vanishes element except that

$$
\begin{aligned}
d_{0,0} & =20 \\
d_{1,0}=d_{-1,0}=d_{0,1}=d_{0,-1} & =-8 \\
d_{1,1}=d_{1,-1}=d_{-1,1}=d_{-1,-1} & =2 \\
d_{2,0}=d_{-2,0}=d_{0,2}=d_{0,-2} & =1 .
\end{aligned}
$$

Then the discrete equation can be written as:

$$
\mathbf{d} * \mathbf{u}=h^{4} .
$$

Applying $\mathcal{F}_{2 n}^{(2)}$ to both side, we obtain

$$
(2 n)^{2} \hat{\mathbf{d}} \bullet \hat{\mathbf{u}}=h^{4} \hat{\mathbf{f}}
$$


where $\hat{\mathbf{d}}, \hat{\mathbf{u}}$ and,$\hat{\mathbf{f}}$ are images under $\mathcal{F}_{2 n}^{(2)}$ of $\mathbf{d}, \mathbf{u}$, and, f respectively, and the images of $\mathbf{d}$ is

$$
\begin{aligned}
\hat{\mathbf{d}} & =\left[20-\left(\cos \left(\frac{k_{1}+k_{2}}{2 n} \pi\right) \cos \left(\frac{k_{1}-k_{2}}{2 n} \pi\right)\right)+\right. \\
& +4\left(\cos \left(\frac{k_{1}+k_{2}}{n} \pi\right) \cos \left(\frac{k_{1}-k_{2}}{n} \pi\right)\right)+ \\
& \left.+8\left(\cos \left(\frac{k_{1}}{n} \pi\right) \cos \left(\frac{k_{2}}{n} \pi\right)\right)\right]_{k_{1}, k_{2}=-\infty}^{\infty} .
\end{aligned}
$$

Multiplying using Hadamard product of $\hat{\mathbf{d}}^{-1}$, we have

$$
\hat{\mathbf{u}}=\frac{h^{4}}{(2 n)^{2}} \hat{\mathbf{d}}^{-1} \bullet \hat{\mathbf{f}}
$$

inverting using 2-dimensional inverse DFT, we get

$$
\mathbf{u}=\frac{h^{4}}{(2 n)^{2}} \mathcal{F}_{2 n}^{(2)-1}\left(\hat{\mathbf{d}}^{-1} \bullet \hat{\mathbf{f}}\right)
$$

and we get the solutions of discrete biharmonic problem

$$
\mathbf{u}=h^{4} \overline{\mathcal{F}}_{2 n}^{(2)}\left(\hat{\mathbf{d}}^{-1} \bullet \hat{\mathbf{f}}\right),
$$

using the fact that

$$
\frac{1}{(2 n)^{2}} \mathcal{F}_{2 n}^{(2)-1}=\overline{\mathcal{F}}_{2 n}^{(2)}
$$

\section{Epilogue}

We have demonstrated an algorithmic development of solving discretization of biperiodic biharmonic equations in unit rectangle. The availability of DFT in several computational platforms such as MATLAB or Scilab, means that the algorithms is easily benchmarked with several necessary additional tools such as Hadamard product, which is unfortunately is not widely available in most computational platforms, hence opening for further work for implementation to be pursued.

\section{References}

[1] M.M. Gupta, Numerical methods for viscous flow problems. In: A.S. Mujumdar(eds.), Advances in Transport Processes, Wiley Eastern, New Delhi, 1980. 
[2] P. Henrici, Fast Fourier methods in computational complex analysis, SIAM Rev. 21 (1979) 481-527.

[3] , Hoang, NS and Damelin, SB, 2017, On surface completion and image inpainting by biharmonic functions: Numerical aspects, arXiv preprint arXiv:1707.06567

[4] I. Sneddon, Elements in Partial Differential Equations, McGraw-Hill, Tokyo, 1984.

[5] G.D. Smith, Numerical Solution of Partial Differential Equations, Oxford Univ. Press, London, 1975. 